\title{
ASRT的
}

\section{PANCS (plantas alimentícias não convencionais): uma abordagem sobre segurança alimentar e educação ambiental em Nova Iguaçu}

\author{
Sandra Maíza dos Santos ${ }^{1}$, Vânia Lúcia de Pádua ${ }^{1}$
}

\section{AUTHOR AFILIATIONS}

1 - Laboratório de Biotecnologia Ambiental; Fundação Centro Universitário Estadual da Zona Oeste. Av. Manuel Caldeira de Alvarenga, 1203 - Campo Grande, Rio de Janeiro - RJ, 23070-200

\section{CONTACT}

vanialucia@uezo.edu.br

sandramaiza26@hotmail.com

\begin{abstract}
This work is constituted as a report of a set of Environmental Education actions developed with high school students in a public school at Nova Iguaçu, periphery of the Rio de Janeiro state. The project was motivated by perception of the socioeconomic vulnerability conditions of the local community, as well as by the quality of life of students and their families. The activities were carried out based on active research methodologies, debates and workshops, aiming at stimulating the participants' self-esteem and empowerment in the face of unfavorable living conditions to which they are exposed, as well as understanding the potential of Nonconventional Edible Plants, and their own backyards, as a natural resource for generating complementary food and even income. It was possible to observe the success of the project throughout the activities, as the students participated actively, gave a positive feedback and generating expectations of changes in eating habits, showing that the construction of knowledge is highly relevant in order to qualify students for a critical posture in the face of socioenvironmental problems, having as perspective the formation of a citizenship that pushes towards the theme of sustainability.
\end{abstract}

Keywords: Environmental Education, Food Security and Nutrition, School; Non-conventional Edible Plants

\section{RESUMO}

Esse trabalho se constitui como um relato de um conjunto de ações de Educação Ambiental desenvolvidas com alunos do Ensino Médio em uma escola pública de Nova Iguaçu, periferia do estado do Rio de Janeiro. O projeto foi motivado pela percepção das condições de vulnerabilidades socioeconômicas da comunidade local, bem como pela qualidade de vida dos alunos e familiares. As atividades foram realizadas com base em metodologias ativas de pesquisa, debates e oficinas, 
objetivando um estímulo à autoestima e ao empoderamento dos participantes diante de condições desfavoráveis de vida às quais estão expostos, assim como o entendimento das potencialidades das plantas Plantas alimentícias não convencionais (PANCs), e de seus próprios quintais, como recurso natural de geração de complementação alimentar e, até de renda. Foi possível observar o sucesso do projeto ao longo das atividades, pois os estudantes participaram de forma ativa, respondendo positivamente e gerando expectativas de mudanças de hábitos alimentares, mostrando que é altamente relevante a construção de conhecimentos visando qualificar alunos para uma postura crítica diante da problemática socioambiental, tendo como perspectiva a formação de uma cidadania que impulsione em direção ao tema da sustentabilidade.

Palavras-chave: Educação Ambiental; Segurança Alimentar e Nutricional; Escola; Plantas Alimentícias Não Convencionais; PANCs

\section{INTRODUÇÃO}

Esse trabalho se constitui como relato de um projeto de Educação Ambiental (EA) desenvolvido com alunos do segundo ano do Ensino Médio de uma escola pública estadual inserida no bairro Marapicu, na cidade de Nova Iguaçu, no estado do Rio de Janeiro.

Paulo Freire (2005) afirma que a libertação pelo processo educacional é válida se for um esforço coletivo, porque as pessoas não se libertam sozinhas, mas sim quando há coparticipação, em comunhão social. A escola é um espaço coletivo e que se constitui como importante agente de mudança com capacidade para promover a transformações locais. As vivências adquiridas ao longo dos anos trabalhando nessa e em outras escolas da mesma região permitiram observar, que parecem valorizar alimentos pouco saudáveis ultraprocessados, além de pouco diversificados, embora tenham quintais e outros espaços disponíveis para plantação, o que favoreceria a adoção hábitos de alimentação mais saudáveis. Além disso, observam-se empreendimentos habitacionais antigos e novos complementando a composição da paisagem do local, além de bois, cabras, charretes e animais silvestres transitando livremente. Há dificuldade em se determinar o que seria rural e urbano, uma vez que o território faz parte de uma zona urbana e periférica da 
cidade. O Plano Diretor da cidade de Nova Iguaçu, publicado em 2011, categorizou o seu território em o espaço público, ambiente urbano e rural. Vale ressaltar que a ruralidade da região foi importante para seu desenvolvimento. Os antigos laranjais que dominam a região de "Iguassu" agora dão espaços a loteamentos e estradas (SIMÕES, 2007, p. 137). Fato é que a cidade é

rica em diversidade e diferentes paisagens

contrastantes, como a paisagem rural, característica dos arredores da escola em Marapicu e a urbana, no bairro Centro, por exemplo.

O espaço escolar promove ensino formal, assim como o não formal, como parte de processos através dos quais as sociedades podem desenvolver suas potencialidades, comportamentos e atitudes, com o propósito de favorecer práticas que privilegiem transformações e participações em tomadas de decisão em prol da sustentabilidade do meio ambiente, conforme proposto no documento Agenda 21. Estas abordagens educacionais buscam a promoção do espírito reflexivo e a transformação do pensamento, que deverá contribuir para mobilizações que visam buscar melhor qualidade de vida. Mas a qualidade de vida é um conceito multidimensional e está relacionada à satisfação do cidadão, no que diz respeito ao acesso à alimentação, saúde e segurança, educação, condições no trabalho, ao lazer e cultura, além de conceitos ligados a uma visão de desenvolvimento sustentável, que têm despontado no cenário da pesquisa sobre indicadores de qualidade de vida (MARTINS, 2002). Dados da ONU informam que é difícil a aquisição de alimentos saudáveis em nossas cidades e a acessibilidade a dietas mais saudáveis precisa melhorar, já que quase um bilhão de pessoas no mundo passam fome ou estão desnutridas e outros dois bilhões têm problemas de saúde decorrentes da má alimentação (FAO, 2006).

A criticidade e os saberes proporcionados pela EA podem favorecer à percepção do aluno e seus familiares quanto à relevância da escolha por alimentos saudáveis, com reduzida contaminação por parasitos, livres de agrotóxicos e outros 
aditivos químicos e com diversidade de nutrientes, além de maior acessibilidade. Esta pode ser alcançada com o consumo de PANCs ou Plantas Alimentícias Não Convencionais. As PANCs são também conhecidas como plantas tradicionais, porque integram um arcabouço cultural composto por tradições indígenas, afrobrasileiras e descendentes de imigrantes, compõem o caldeirão cultural brasileiro. Os avanços e interesses da indústria alimentícia culminaram por ocultar conhecimentos tão antigos como as propriedades, sabores e potencialidades das plantas tradicionais ou "mato de comer" (KINUPP, LORENZI, 2014). No contexto de crescimento da hegemonia da indústria alimentícia foi sendo edificada uma cultura contra os saberes tradicionais e um preconceito foi se arraigando em relação ao cultivo e consumo das plantas tradicionais, e boa parte delas caíram no esquecimento. Porém, algumas se mantiveram presentes devido à resistência e permanência teimosa de algumas tradições regionais que se utilizam de seus sabores e aromas em seus pratos típicos, e pela tradição, principalmente, indígena e afro- brasileira que mantém vivos alguns costumes e ensinamentos dos antepassados que são transmitidos de geração em geração. $\mathrm{O}$ cultivo de muitas dessas plantas tem ressurgido em uma perspectiva de valoração do natural e do tradicional, especialmente no que se refere a alimentação saudável por meio de hortaliças, frutas, raízes, frutos, sementes e flores, outrora vistas como exóticas, hoje exploradas pelo valor nutricional e/ou propriedades medicinais (KINUPP, LORENZI, 2014). O incentivo ao consumo de alimentos saudáveis, alternativos, acessível e com baixo custo como as PANCs pode se configurar como um instrumento para promoção de transformações positivas, além de permitir o estabelecimento da conexão das pessoas, antes tão familiarizadas com o consumo de alimentos industrializados, com os fundamentos da alimentação.

A constatação da dura realidade local motivou a idealização deste trabalho, com o objetivo de expandir o entendimento sobre a comunidade local e promover uma conscientização ambiental nas famílias, a partir 
de pesquisas e vivências dos alunos no tocante à sua alimentação, meio ambiente, visando contribuir para melhorias na sua qualidade de vida, no que concerne alimentação, meio ambiente e oportunidade de geração de renda, pelo reconhecimento das suas potencialidades. Nessa perspectiva, espera-se contribuir para transformação de atitudes e ainda, estimular um sentimento de pertencimento ao seu próprio meio, algo importante para autoidentidade e autovalorização, favorecendo também a

formação de corresponsabilidades pela preservação e uso sustentável dos recursos naturais (SCHNEIDER, 2013).

\section{METODOLOGIA}

O presente estudo é fruto das experiências de oficinas realizadas em 2019 com alunos do Centro Integrado de Educação Pública - CIEP 354 Martins Pena, escola pública estadual inserida no bairro Marapicu, na cidade de Nova Iguaçu, no estado do Rio de Janeiro. Uma das autoras é professora nesta escola. Participaram da oficina 70 alunos da segunda série do Ensino Médio, com
16 anos, em média. Os alunos foram distribuídos em quatro grupos, de modo que pudessem interagir melhor com o objeto de conhecimento em questão, seus pares e a docente. O estudo foi feito com alunos do Ensino Médio por estarem em uma faixa etária propícia para o desenvolvimento de reflexões críticas e responsáveis e para construir valores condizentes com novas e pertinentes atitudes e práticas propostas relacionadas ao consumo, autocuidado, valorização do outro e do meio ambiente.

O Referencial Teórico da pesquisa foi fundamentado no rastreamento de dados nacionais e internacionais derivados de artigos científicos coletados da base dados do Google Acadêmico, sítios eletrônicos de instituições governamentais e não governamentais, relacionados à região estudada, Indicadores Sociais Municipais, Educação Ambiental e PANCs.

A partir da pesquisa acima, foram selecionados os trabalhos utilizados como principal base teórica na oficina. Foram eleitos os trabalhos de Kinupp e Lorenzi (2014) e Madeira 
(2013) ambos de 2014 sobre as PANCs, de Pelicioni (1998) sobre EA e trechos do Atlas escolar da cidade de Nova Iguaçu (2004). Em seguida, foram iniciadas as propostas de atividades pedagógicas na escola campo de pesquisa.

O estudo também foi baseado em dados de cunho empírico, envolvendo percepção e conhecimento dos alunos de ensino médio da escola supramencionada, submetendo-os a um questionário exploratório e a quatro diferentes etapas relacionadas à oficina, ocorridas em momento diferentes. A oficina teve quatro horas de duração e alguns dos seus momentos incluindo local de coleta, preparação de alimento no refeitório e o debate com os alunos são mostrados na figura 1.

$1^{\circ}$ Etapa: Foram realizadas palestras sobre o tema relatando sobre o conceito de PANCs, suas propriedades, os regionalismos e tradições que as envolvem, além da aplicação na culinária e na medicina natural. Durante esta etapa a metodologia foi baseada na exposição dialogada e leitura comentada de textos apresentados sobre
EA, aspectos de zona rural e urbana de Nova

Iguaçu, horta e alimentos PANCs; individualmente e em grupo. Os estudos foram sistematizados pelos alunos por meio da produção de cartazes e dos debates em sala de aula.

$2^{\circ}$ Etapa: Os alunos puderam responder às perguntas relacionadas conceitos de EA, entendimento pessoal de cada um sobre alimentação saudável, escolhas rotineiras para a principal refeição (almoço), se possuem uma horta em casa e também, o conhecimento sobre hortaliças PANCs e seu potencial alimentício.

$3^{\text {a }}$ Etapa: Execução da oficina sobre PANCs no refeitório. Para a realização da oficina foram utilizados como materiais folhas de caderno para anotações, água limpa e utensílios para preparação dos alimentos (panelas, talheres e pratos), que foram disponibilizados pela escola. Os demais materiais utilizados na oficina foram coletados através de uma campanha feita pela professora, co-autora deste trabalho, com a participação de alunos e da escola, que forneceu parte dos alimentos que já seriam usados na alimentação dos próprios alunos. A hortaliça 
Peixinho foi levada pela professora, demais hortaliças como Caruru e Taioba foram coletadas no terreno do entorno da escola, junto dos alunos.

No campo os alunos desenharam ou tiraram foto com seus celulares pessoais. Em sala de alua, tiveram contato direto com os exemplares das PANCs dispostos sobre as mesas com seus nomes e a região brasileira de origem, e/ou onde são mais conhecidas e/ou consumidas. $\mathrm{Na}$ sequência, os alunos foram divididos em grupos, e a cada grupo foi entregue um exemplar de PANC e uma receita de possibilidade de utilização alimentícia desse exemplar. Cada grupo preparou um alimento, e ao final, todos sentaram-se à mesa para saborear o resultado dessa experiência.

Durante a oficina foram trabalhados os cuidados com o manejo das plantas, os possíveis usos, as suas propriedades nutricionais e potenciais benefícios na alimentação cotidiana. Em paralelo, foram abordadas formas de utilização de algumas mudas, visando demonstrar que havendo espaço em casa, como um quintal, é possível cada um ter a sua própria horta, adaptada a realidade de cada um.

$4^{\mathrm{a}}$ Etapa: Os alunos foram questionados visando analisar as experiências pedagógicas vivenciadas na oficina, com a exposição dos pontos positivos. A atividade foi finalizada com um exercício de reflexão e expressão oral sobre as dificuldades relativas às necessidades para obtenção de qualidade de vida, saúde e renda dos alunos, seus familiares e a comunidade. Em seguida, foi pedido aos alunos que pensassem e propusessem soluções para os problemas apontados por eles, envolvendo as PANCs. 


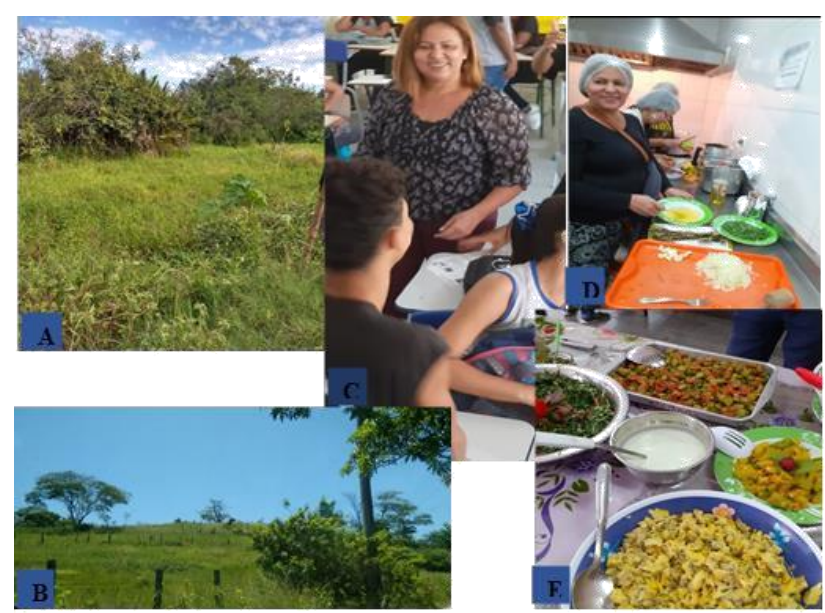

Figura 1: oficina sobre pancs. A e b - regiões no entorno da escola. ; c - a professora e seus alunos em sala de aula, debatendo sobre o tema; d - preparação da refeição; ealgumas das refeições com pancs, preparadas junto dos alunos.

\section{RESULTADOS E DISCUSSÕES}

O estado do Rio de Janeiro é marcado por realidades contrastantes e a cidade de Nova Iguaçu está entre as que possuem alguns dos piores indicadores de pobreza e renda do Estado, reunindo muitas famílias em situação de

vulnerabilidade ambiental (ROCHA \& ALBUQUERQUE, 2007). A região está localizada na periferia e apresenta alguns dos piores indicadores sociais e de qualidade de vida na Região Metropolitana (IBGE, 2019). Ponderar sobre qualidade de vida remete ao Índice de Desenvolvimento Humano Municipal (IDHM), que pontua 0,713 na cidade de Nova Iguaçu. A incidência de pobreza a posiciona como $8^{\circ}$ mais pobre, dentre 91 cidades (IBGE, 2019). A região tem problemas que dificultam o desenvolvimento de programas de saúde, muitas vezes em decorrência da pouca segurança observada, ampliada pela disputa de território pelo tráfico e

milícia (Rocha, 2020 p. 20). Um desenvolvimento pleno requer infraestrutura adequada, incluindo itens que favorecem melhores condições de vida e a redução de desigualdade, considerando saúde, alimentação, meio ambiente e educação (BELTRÃO \& SUGAHARA, 2005).

Este trabalho foi idealizado a partir de inquietações surgidas durante vivências prévias experimentadas entre alunos de pelo menos duas escolas públicas da região, além da comunidade local, que apontaram uma alimentação pouco diversificada e com lanches baseados preferencialmente em alimentos ultraprocessados e um baixo aproveitamento de recursos 
disponíveis, como os quintais de suas casas para plantação de horta, sugerindo a necessidade de intervenção em amplitude coletiva sobre práticas de sustentabilidade e melhoria da qualidade de vida.

O conceito de desenvolvimento sustentável é definido no relatório intitulado Nosso Futuro Comum (BRUNDTLAND, 1987), elaborado pela Comissão Mundial sobre o Meio Ambiente e Desenvolvimento, como aquele que "atende às necessidades do presente sem comprometer a capacidade das gerações futuras atenderem às suas". Existem três elementos importantes para que o desenvolvimento sustentável seja alcançado: crescimento econômico, inclusão social e proteção ao meio ambiente. Esses elementos são interligados e devem estar harmonizados, visando o alcance de uma vida digna, o bem-estar dos indivíduos e das sociedades.

Em 2015, chefes de Estado e altos representantes da Organização das Nações Unidas reuniram-se e adotaram os Objetivos de Desenvolvimento Sustentável (ODS) que propõem uma ação mundial coordenada entre os governos, as empresas, a academia e a sociedade civil para alcançar 17 ODS e suas 169 metas, visando a promoção de vida digna para todos (ONU BRASIL). As ODS 2 e 3, relacionam-se às questões de segurança e soberania alimentar, abordando a fome zero, agricultura sustentável, boa saúde e bem-estar.

No Brasil, a Lei Orgânica de Segurança Alimentar e Nutricional (LOSAN), Lei $\mathrm{n}^{\circ}$ 11.346 de 2006, tem como objetivo a garantia da segurança alimentar e nutricional. Esta consiste na realização do direito de todos ao acesso regular e permanente a alimentos de qualidade e em quantidade suficiente, sem comprometer o acesso a outras necessidades essenciais, tendo como base práticas alimentares promotoras da saúde, que respeitem a diversidade cultural e que sejam ambiental, cultural, econômica e socialmente sustentáveis.

Uma das soluções que vem sendo propostas para essa questão é a agricultura familiar, que corresponde à produção agrícola desenvolvida por famílias e voltada para a 
subsistência das mesmas, muitas vezes ocorrendo nos próprios quintais de suas casas. A agricultura familiar pode garantir a produção de alguns de seus alimentos e, em alguns casos, ser uma oportunidade de geração de renda. Além disso, reconectando as pessoas com a comida de verdade, ao invés do ultraprocessados, as famílias se alimentam com melhor qualidade, pela nutrição e por serem livres de agrotóxicos, com chances para ampliação da convivência em comunidade. É fundamental que a população se conscientize sobre a importância de uma alimentação saudável e diversa, já que existem sérias implicações sobre a probabilidade de desenvolver problemas de saúde em consequência.

Este trabalho trata de uma ação de Educação Ambiental em uma escola pública de Nova Iguaçu. A ação aconteceu em encontros semanais e incluiu explanação teórica, debates e um momento prático de oficina culinária. O projeto foi apresentado pela professora co-autora e obteve total aceitação por parte dos gestores da escola e demais atores envolvidos, que consideravam necessárias as abordagens de sensibilização para mudança de hábitos alimentares como estratégia de segurança alimentar e nutricional, bem como contribuição para o alcance de alguns dos 17 Objetivos de Desenvolvimento Sustentável.

Inicialmente, os alunos ouviram a palestra e leram em grupo sobre EA e a cidade de Nova Iguaçu que possui zonas rurais e urbanas, relacionando o desenvolvimento sustentável ao autocuidado e a preservação do meio ambiente, e finalmente, sobre a boa alimentação, com foco em vegetais e nas PANCs, exemplificando alguns tipos, aplicações culinárias e medicinais. $\mathrm{Na}$ sequência, foi colocado que as PANCs são encontradas no entorno da escola e talvez em suas casas e que podem ser plantadas em uma horta feita em casa, assim como outros vegetais. Ainda nesta etapa alguns dos alunos fizeram cartazes sobre EA e/ou PANCs e questionaram um pouco sobre tudo que foi apresentado, sendo que a maioria relatou sobre suas habilidades para preparações culinárias, práticas que favorecem o 
desenvolvimento sustentável e a falta de conhecimento sobre alimentação saudável.

Uma das informações pesquisadas pelos alunos que mais chamou atenção dos mesmos foi sobre o fato de existirem no mundo mais de 30 mil espécies de plantas comestíveis. Dentre estas, cerca de 7 mil já foram utilizadas na nossa história. Mesmo assim, atualmente, $90 \%$ do alimento da população mundial vem, principalmente, de cerca 20 espécies de plantas (Parque das Aves, 2020), demonstrando que consumimos somente uma pequena parcela da biodiversidade existente.

Na segunda etapa os alunos responderam ao questionamento sobre seus conceitos de EA, especialmente relacionada à alimentação. As respostas, em sua maioria, descreviam a sua própria condição de alimentação e mostrou uma conduta passiva em relação aos próprios problemas ou, na maioria das vezes, invisibilidade do problema, como o frequente consumo de ultraprocessados no lanche e outras refeições.
As respostas sobre seus entendimentos sobre alimentação saudável foram respondidas com exemplo de cardápio, de modo que $60 \%$ responderam que saudável é comer verduras e legumes, $20 \%$ responderam que é não comer frituras e os demais $20 \%$, não comer muito sal e açúcar. Quando questionados sobre hábitos alimentares na hora do almoço, todos responderam que comem a mistura de arroz e feijão todos os dias, sendo que $40 \%$ dos alunos adicionam ovo a este prato. Os demais $60 \%$ ficaram mais ou menos igualmente distribuídos entre frango, legumes e "algum tipo de carne", além do arroz e feijão. Embora este cardápio tipicamente brasileiro tenha uma boa combinação de nutrientes e seja geralmente acessível para a sociedade, é importante adicionar vegetais e

ampliar a diversidade de nutrientes e a quantidade de fibras, visando uma alimentação adequada (SICHIERI et al., 2000). O consumo de hortaliças acessíveis como as PANCs preenche bem esses requisitos.

Os alunos foram questionados se possuem uma horta em casa e, embora a grande maioria tenha residência em casa com quintal, apenas 30 
$\%$ responderam positivamente. Este resultado motivou a ampliação do debate sobre EA através da horta do quintal. Quando questionados sobre seu conhecimento sobre as PANCs e seu potencial alimentício, apenas um número reduzido, perto de $10 \%$ dos alunos, respondeu positivamente. Mas estes, em sua maioria, na verdade, já conheciam alguns exemplares de PANCs, como a taioba, ora-pro-nobis e a bertalha, sem atribuir a designação, nem reconhecer sua importância. Para a maioria dos alunos algumas das PANCs apresentadas eram conhecidas como mato, porém nunca visualizadas como alimento, como por exemplo, o Caruru, planta muito comum na região, e cujas folhas, sementes e flores podem ser consumidas como alimento, embora sempre cozidas.

A oficina foi realizada com participação ativa dos alunos, desde a coleta até a preparação da comida e finalmente, a degustação. Durante a coleta foram transmitidos conceitos sobre seu manejo e as propriedades das plantas usadas para preparação, que foram Peixinho da Horta, Caruru e Vinagreira. Além de fonte de fibras, são ricas em minerais e podem ser usadas tanto na culinária quanto na terapêutica popular, conforme descrito em KINUPP \& LORENZI (2014). Foram escolhidas receitas que além de saudáveis eram simples, saborosas e de baixo custo, Peixinho da Horta servido empanado frito ou em omelete, salada de Vinagreira e também Caruru ensopado com angu. A aceitação dos pratos foi muito boa.

Foram marcantes os momentos de descontração e interação entre todos os presentes, o que favoreceu a participação ativa dos participantes e o entendimento da oficina como um estímulo à convivência sustentável, bem como uma estratégia bem sucedida de reconexão cultural e ambiental com a natureza. A grande maioria se dedicou com afinco às coletas e aprovou as preparações, colocando que tentaria reproduzir em casa. Os grupos de educandos apresentaram suas conclusões e descobertas, relatando valores nutricionais e propriedades medicinais com entusiasmo, principalmente por ser, ao mesmo tempo que uma grande novidade, também um elemento antigo, pois eram plantas que desde sempre estiveram presentes em seus quintais, ou em sua vizinhança. 
A escola pode ser enxergada como um reflexo da sociedade por estudiosos como Bourdieu \& Passeron (1975) e tem sido descrita como a referência para a vida dos estudantes no mundo contemporâneo, sustentando a organização da sociedade em geral (CAVALIERE, 2007). Assim, os resultados observados parecem indicar a relação da comunidade local com o meio ambiente. A atividade estimulou a elaboração de um projeto de Horta na Escola e na semana seguinte os grupos planejaram fazer novas coletas, com foco nas espécies de presentes em seus próprios quintais para em seguida identificá-las como PANCs, tomarem ciência sobre cuidados básicos para evitar o risco de intoxicações; e finalmente decidirem quais poderiam ser utilizadas em outra oficina similar. Os planos ficaram pendentes até a plena recuperação das condições de segurança de saúde nos ambientes da escola, quanto à propagação do Sars-CoV-2, causador da pandemia da Covid-19.

Finalmente, na última etapa os alunos levaram as suas reflexões sobre a oficina e revelaram outros pontos positivos. Levaram relatos de seus familiares e vizinhos relacionados ao consumo e cultivo de algumas dessas plantas, observando que em alguns casos estavam intimamente ligados a histórias tradicionais e com aspectos culturais, tendo em vista que muitos desses familiares e vizinhos eram oriundos de outras regiões do Brasil. Em seus relatos, os familiares haviam contado histórias sobre como utilizavam as PANCs nas comidas de seus lugares de origem, e que haviam aprendido sobre seus sabores, propriedades e usos com suas mães, avós, mas com o tempo estes hábitos haviam se perdido. De fato observa-se há uma crescente diminuição no consumo de hortaliças no Brasil em decorrência da "globalização e do crescente uso de alimentos industrializados, em consequência de mudanças significativas no estilo de vida e no hábito alimentar dos brasileiros" (MADEIRA et. al. 2013). Os prejuízos são ainda maiores considerando que este fato pode contribuir para perdas no tocante às histórias e referenciais culturais, e mais especificamente, com relação às hortaliças tradicionais, a redução no cultivo e consumo vem 
ocorrendo pela perda da referência da produção local (MADEIRA et. al. 2013).

Em seguida, foi pedido aos alunos que pensassem e propusessem soluções para os problemas apontados por eles, envolvendo as PANCs. Sintetizando todas as falas, os alunos foram introduzidos em conceitos de segurança alimentar e nutricional, houve percepção sobre a importância da qualidade da alimentação para obtenção de saúde e qualidade de vida e de que PANCs são alimentos que têm sido

desperdiçados. Interessante, os alunos enxergaram possibilidades para seu empoderamento, similar ao já descrito em Vieira et al. (2018). Além disso, levando em consideração o potencial local, as PANCs e outras plantas cultivadas em horta podem também se tornar uma possibilidade de geração de renda para famílias de baixa renda, de modo sustentável, a partir de plantio e coleta sistematizados, do incentivo ao seu consumo, e de sua comercialização, contemplando uma prática humana consciente e "responsável pela busca de equilíbrio relativo entre as necessidades básicas dos grupos sociais e os recursos do meio natural" (KINUPP; LORENZI, 2014).

\section{CONCLUSÕES}

O presente trabalho contribuiu para as reflexões de educandos sobre suas condições de vida, bem como as de seus vizinhos e familiares, sobre a necessidade de se pensar em possibilidades de melhorias de qualidade de vida e na alimentação, de si mesmo e para a coletividade. Desse modo, as atividades realizadas e os conhecimentos construídos a partir de seu desenvolvimento se configuraram como um estímulo à autoestima e ao empoderamento dos participantes diante de condições desfavoráveis de vida às quais estão expostos, $\mathrm{e}$

sua percepção sobre as potencialidades das plantas tradicionais, assim como de seus próprios quintais como recurso natural de geração de complementação alimentar, e até de renda. A escola pode ser um espelho da sociedade e uma amostra dos estudantes de uma escola pública local revelou que esta sociedade parece alheia sobre a profundidade de alguns de seus problemas e potenciais. As informações obtidas apontam 
para a importância da construção e disseminação de conhecimentos visando qualificar alunos para uma postura crítica diante da problemática socioambiental, tendo como perspectiva a transformação de hábitos e práticas sociais que os impulsione em direção ao tema da sustentabilidade, no seu sentido mais amplo.

\section{Agradecimentos}

A todos aqueles que contribuíram, de alguma forma para a realização deste trabalho e às instituições de ensino e pesquisa do Estado do Rio de Janeiro participantes, especialmente à UEZO e a FAPERJ.

\section{REFERÊNCIAS}

Atlas escolar da cidade de Nova Iguaçu Site da Prefeitura de Nova Iguaçu. 2004. Disponível em: http://www.novaiguacu.rj.gov.br/arquivos/atlase scolar.pdf. Acesso em 17 de Ago. de 2019.

BELTRÃO, K. I.; SUGAHARA, S. Infraestrutura dos domicílios brasileiros: uma análise para o período 1981-2002. Rio de Janeiro: IPEA, Texto para discussão, n. 1077, 2005.
BOURDIEU, P.; PASSERON, J. A Reprodução: elementos para uma teoria do sistema de ensino. [Trad. Reynaldo Bairão]. Rio de Janeiro: Francisco Alves Editora S/A (Série Educação em Questão, 1975.

BRASIL. Lei $\mathrm{N}^{\circ} 11.346$ de 15 set. 2006. Lei Orgânica de Segurança Alimentar e Nutricional (LOSAN). Cria o Sistema Nacional de Segurança Alimentar e Nutricional - SISAN com vistas em assegurar o direito humano à alimentação adequada e dá outras providências. Diário Oficial da União. 18 set 2006. Acesso em 22 de dez 2020. Disponível

em: https://www.planalto.gov.br/ccivil_03/_ato 2004-2006/2006/lei/111346.htm.

BRUNDTLAND, Gro Harlem — “Our Common Future - The World Commission on Environment and Development" - Oxford University, Oxford University Press, 1987.

CAVAliERE, Ana Maria. Tempo de escola e qualidade na educação pública. Educ. Soc., Campinas , v. 28, n. 100, p. 1015-1035, Oct.

2007. Available from <http://www.scielo.br/scielo.php?script=sci_artt ext\&pid=S0101$73302007000300018 \& \operatorname{lng}=e n \& n r m=i s o>$. access on 17 Jan. 2020. 
FOOD

AND

AGRICULTURE

ORGANIZATION (FAO), WORLD HEALTH

ORGANIZATION (WHO). Food safety risk

analysis. A guide for national food safety authorities. Rome: FAO; 2006. Disponível em: https://apps.who.int/iris/bitstream/handle/10665/ $\underline{43718 / 9789251056042 \text { eng.pdf?sequence }=1 \& \text { is }}$

Allowed=y. Acesso em: 22 out. 2020.

KINUPP, Valdely Ferreira; LORENZI, Harri. Plantas alimentícias não convencionais: guia de identificação, aspectos nutricionais e receitas ilustradas. São Paulo: Inst. Plantarum de Estudos da Flora, 2014.

MADEIRA, Nuno Rodrigo. et. al. Manual de produção de hortaliças tradicionais. Brasília: EMBRAPA, 2013.

MARTINS C. H. B. Pobreza, meio ambiente e qualidade de vida: indicadores para o desenvolvimento humano sustentável. Indic. Econ. FEE, Porto Alegre, v 30, n. 3, p 171-188, $\operatorname{dez} 2002$.

NOVA IGUAÇU. LeiEI Nº. 4.092, deDE 28 deDE junhoJUNHO de DE 2011. "Institui o
Plano Diretor Participativo e o sistema de gestão integrada e participativa da cidade de Nova Iguaçu, nos termos do artigo 182 da Constituição Federal, do capítulo III da Lei N$^{\circ} 10.257$, de 10 de julho de 2001 - Estatuto da cidade e do art. 14, § $3^{\circ}$ da lei orgânica da cidade de NOVA

IGUAÇU” Disponível em

https://www.cmni.rj.gov.br/site/legislacao-

municipal/plano-diretor/plano-diretor-

republicacao-em-06-06-12-2-31.pdf Acesso 17

de ago de 2019.

ONU BRASIL. Objetivos de desenvolvimento sustentável: 17 objetivos para transformar nosso mundo. Brasília, 2015. Disponível em: https://brasil.un.org/pt-br/sdgs. Acesso em: 30 mar. 2019.

PARQUE DAS AVES. Conheça tudo sobre PANCs. (2020). Disponível em: <https://blog.parquedasaves.com.br/2020/01/pan c-mata-

atlantica/\#: :text=No\%20mundo $\% 20$ todo $\% 2 \mathrm{C} \%$ 20estima\%2Dse, apenas\%2020\%20esp\%C3\%A9 cies\%20de\%20plantas!>. Acesso em 22 ago. 2020. 
PELICIONI, Maria Cecília Focesi. Educação ambiental, qualidade de vida e sustentabilidade. Saúde Soc., São Paulo, v. 7, n. 2, p. 19-31, dez. 1998.

ROCHA, André Santos. Baixada Fluminense: estudos contemporâneos e (re)descobertas históricogeográficas. A violência e o descaso social - a representação hegemônica ainda persiste. Organizado por André Santos da Rocha - Duque de Caxias: ASAMIH. 2020, 315p.

Disponível em

https://d1wqtxts1xzle7.cloudfront.net/63573643/ $\underline{\text { Livro_- }}$

_Baixada_Fluminense_2020_FINAL_OKcapa20 $\underline{200609-90714-}$

$\underline{117 \mathrm{ktdn} . p d f ? 1591704877=\& \text { response-content- }}$ disposition=inline $\% 3 \mathrm{~B}+$ filename $\% 3 \mathrm{DBAIXAD}$

A_FLUMINENSE_estudos_contemporaneo.pdf $\underline{\& \text { Expires }=1610122870 \& \text { Signature }=\text { ZB- }}$

GOSEPLoqLWeHyN5bObxas-

12oCQt3EsOG2a1ak6F68EYht9hkbruDrYgMEE cBUIe435Mqm2ZG Dg5E7B-

N911Hb9PQpMfIfuaktriJJOn Ha5CiLgSXKZqS $\underline{9 m-C O 22-}$
qMcHDAvmnKeIDSWDyuHPRD4wbTpdYuoV

SJTFBZdSpgJ3RtXJPkKMTVBcFILy3-

$\underline{\text { 1sgakJ1I11TDWBV5vVyGbpp0zkKtKNYqSUe }}$

UFVahQCYnCcQHr0s6UP8Mjcxjdv9-

5RIO6d1PWDwFf8mUDylFu6g3Gsth2oawvIB

$\underline{x}$

$\underline{\text { PZO6k389PAG QZ8ZrCHHS C5nWCu9DhjH }}$

$\underline{\text { MSMvGSBw } \sim \text { wP6ve5Jg_\&Key-Pair- }}$

$\underline{\text { Id=APKAJLOHF5GGSLRBV4ZA\#page=14. }}$.

Acesso 2018 de setembro dezde 202019.

SCHNEIDER, S. Educação do Campo e Sustentabilidade: $\mathrm{O}$ caso da Escola família Agrícola em Santa Cruz do Sul - RS. Atos de Pesquisa em Educação, 8(3), 964-985, 2013.

SICHIERI, Rosely et al . Recomendações de alimentação e nutrição saudável para a população brasileira. Arq Bras Endocrinol Metab, São Paulo, v. 44, n. 3, p. 227-232, June 2000.

Available from <http://www.scielo.br/scielo.php?script=sci_artt ext\&pid=S0004-

$27302000000300007 \& \operatorname{lng}=\mathrm{en} \& \mathrm{nrm}=\mathrm{iso}>$.

Accesso em 07 de janeiro de 2021.

https://doi.org/10.1590/S0004-

$\underline{27302000000300007}$ 
SIMÕES, Manoel Ricardo. A cidade estilhaçada:

reestruturação econômica e emancipações

municipais na baixada fluminense. Mesquita: ed.

Entorno, 2007.

VIEIRA, Márcia Gilmara Marian. et. al.

Educação para transformação: Empoderamento

feminino alicerçado nos princípios da agroecologia. Ambiente: Gestão e

Desenvolvimento,[S. l.], v. 11, n. 01, p. 167-192,

2018. DOI: 10.24979/158. Disponível em:

https://periodicos.uerr.edu.br/index.php/ambient

e/article/view/158. Acesso em: 19 ago. 2020. 